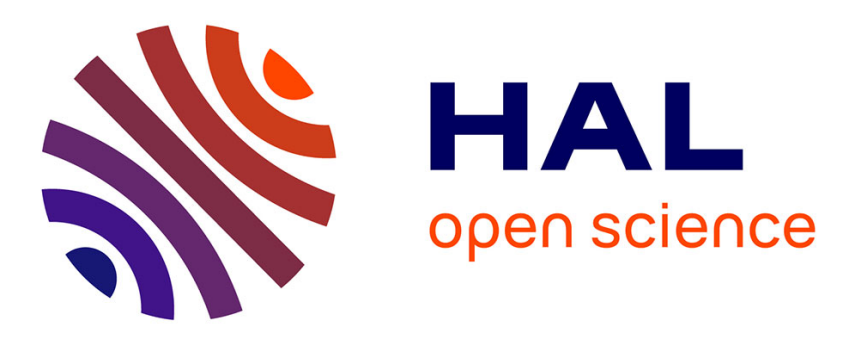

\title{
Physical modelling for interactive installations and the performing arts
}

\author{
Sarah Fdili Alaoui, Cyrille Henry, Christian Jacquemin
}

\section{To cite this version:}

Sarah Fdili Alaoui, Cyrille Henry, Christian Jacquemin. Physical modelling for interactive installations and the performing arts. International Journal of Performance Arts and Digital Media, 2014, 10 (2), pp.159 - 178. 10.1080/14794713.2014.946284. hal-01663115

\section{HAL Id: hal-01663115 https://hal.inria.fr/hal-01663115}

Submitted on 21 Dec 2017

HAL is a multi-disciplinary open access archive for the deposit and dissemination of scientific research documents, whether they are published or not. The documents may come from teaching and research institutions in France or abroad, or from public or private research centers.
L'archive ouverte pluridisciplinaire HAL, est destinée au dépôt et à la diffusion de documents scientifiques de niveau recherche, publiés ou non, émanant des établissements d'enseignement et de recherche français ou étrangers, des laboratoires publics ou privés. 


\title{
Physical Modelling for Interactive Installations and the Performing Arts
}

\author{
Sarah Fdili Alaoui, Cyrille Henry, and Christian Jacquemin
}

\begin{abstract}
This article explores new artistic possibilities through four different examples of digital environments where live performance and virtual physical models come together. Physical models (specifically mass-spring systems) are used to enhance mappings between human movement and digital rendering. In the case of multi-modal rendering, physical models improve the cross-modal coherence. Given a physical model's capacity to simulate physical behaviors, they can also be used to visualise or to simulate human movement qualities, and even to simulate a dancer's virtual partner. More applications certainly remain to be tested, but the present study opens new avenues for the exploration of the complex and physically plausible behaviors of mass-spring systems in the context of both artistic practice and art pedagogy.
\end{abstract}

\section{Keywords}

Computer Graphics; Augmented Performance; Digital Scenography; Physical Models; Mass-Spring Systems. 


\section{Introduction}

The introduction of 'new' media into contemporary performing arts is not a new phenomenon'. The digital era has opened new and responsive possibilities, which are anchored in collaborative entanglements between performing arts, design and engineering. The design of interactive environments controlled by movement and other expressive body channels have been developed along various dimensions: control of sound composition (David Rokeby's Very Nervous System, 1991), synthesis of visuals according to vocal expression (Golan Levin and collaborators' Messa di Voce, 2003), revelation of videos in a monumental installation (Lozano-Hemmer's installation Body Movies, 2001), and related to dance in particular, they include the transformation of physical movements into 3D projections (Kim Vincs and John McCormick Aura's augmented ballet, 2009).

The appeal for enhanced interactivity has led artists to search for more complex models with which to create virtual performers (e.g. Merce Cunningham's Biped, John Crawford and Martin Gotfrit's Active Space + Embodied Media) or physical models (e.g. those developed for cinematic art by Chi-Min Hsieh and Annie Luciani, 2005). In this article, we will investigate how digital simulation of physical models can be used for live performance. Physical models have an important expressive potential but present a drawback: they are difficult to control because of their complex dynamics and the high dimensionality of their parameter space. In the context of live performance, such models have to be adapted in order to allow a performer to control them while being dedicated to his/her expressiveness instead of coping with sophisticated control interfaces.

This article focuses on a specific family of physical models, namely mass-spring systems (MSS), and proposes four illustrative applications of the adaptation of these models to performance and artistic training in which the relationship between artistic expression and technological issues is studied in detail:

1. an audio application for a dance performance in which a MSS controls sound parameters and gives physicality to the sound,

2. a visual application for dance pedagogy in which a MSS reflects the dancer's movement qualities and gives him/her a visual feedback about his/her performance of these qualities,

3. a visual application for an augmented dance performance in which a massive MSS made of thousands of masses creates an interactive scenography,

4. an audio-visual performance in which both sounds and images are a direct representation of a MSS behaviour.

Through a collaborative design process involving both scientists and artists, we explore how the physicality of these physical models evokes expressive capabilities and supports the performer's engagement through embodied interaction in interactive environments within live performance. Illustrative documentation of the performances and the collaboration with professional dancers and artists

$1 \quad$ The Nine Evenings, a series of avant-garde live performances and new technologies organized by Robert Rauschenberg and Billy Kluwer in October 1966, together with earlier experiences such as Maurice Bejart's CYSP 1 ballet with Nicolas Shöffer's interactive sculpture in June 1956 have shown how entangled performing arts and interactive media can be, to the point that the digital work has become a full partner in performance design. 
allows us to assess the many directions along which these models can be used in the performing arts. In particular we will investigate the capacities of physical models to generate expressive and physicoplausible behaviours for performance, with real-time control and synthesis for different media such as sound or graphics.

\section{Physical Models: Expressive metaphorical behaviours and control mappings in digital performance environments}

Physical models are complex systems that require heavy computation to be used interactively on stage. It is only recently that standard equipment can offer enough computational power and fast graphical rendering to allow the interactive use of physical models on stage. Klaus Obermaier's Apparition (2004) combines dance with video rendering of interactive physical models, which is connected with the dancers' dynamics. Real-time body silhouette capture is used to selectively project different video renderings on the performers' bodies. In addition to striking visual effects, physical models are also intended to play the role of virtual dancers interacting with actual dancers through the ability to create dynamic and kinesthetic responses. For this reason, the virtual model rendering has to be fluid and responsive to the performer. Fluidity is related to the frame rate of the graphics engine, and is mostly a matter of engineering and optimization whereas responsiveness is the capacity of the system to analyse in real-time the input from the performer and to output dynamic behaviours accordingly. A responsive model should be able to respond to the performer with behaviours that are perceived as a genuine partner with self-agency facilitating a rich dialogue and duet on stage. It should not respond with behaviours that are perceived as a literal mirror of the performer's movement. Physical models require heavy programming in order to achieve satisfying and expressive effects with fluid rendering and real-time responsive behaviours.

Adrien Mondot's project Convergence 1.0 (2005) explores juggling with physical models, although in less complex set-ups than those used in Apparition, in Mondot's work, physical modeling offers fluid visuals with non-realistic physical properties to equip the performer with virtual balls that defy the laws of gravity and adopt autonomous behaviours on-stage. Through real-time capture, the virtual models are responding synchronously to the staged events. The physical models act as a distorted mirror of the actual performance physicality: they can amplify physicality or develop autonomous variations on it.

Winterspace (2001) by Igloo, the collective of Ruth Gibson and Bruno Martelli, represented bodies inside an interactive computer graphics environment using luminous snowflakes that were controlled by the dancers' movements. Following directly from this Kirk Woolford's installation Will.0.W1sp (2006) illustrates a more constrained correlation between the performer's actions and the visual renderings than the two previous examples because the particle system generation is based on preliminary motion capture rather than real-time data input. It reveals an interesting feature of our empathic perception of animated patterns where we are very likely to accept the patterns as stylized human shapes, even if the resemblance between the animated figures and a human silhouette is a distant one. The physicality of the model and its possible similarity with human body dynamics drives the onlooker or the performer towards a 'dialogue' and a mutual play between his/her own gestures and the semi-autonomous behaviours of the virtual model. Both of these works utilise the capacity of our vision making system to rebuild visual figures from partial information such as dots scattered on a surface representing a human or animal shape (Gregory 1970). The use of large-scale physical models to render interactive dots with recognisable plausible behaviors will be further developed in this article with reference to the installation Double Skin/Double 
Mind and the performance Chiseling Bodies. In the latter, the physical models are controlled so that their dynamics can metaphorically evoke the expressive movement qualities of the dancer and differ from the former examples on two important features. Firstly they focus on movement qualities, a qualitative expressive aspect of movement largely explored in dance. Secondly, they do not offer explicit visual feedback to the dancer and to the audience (such as a figure inspired by a human shape) but rather propose an abstract and suggestive rendering of physical models. By doing so, they open new avenues for the use of a much higher quality of interactive visuals in real time performance. More abstract visuals allow for a wider range of conceptual responses than visuals obtained from motion capture or image processing. Interviews with performers and on-line experiments showed the keen interest of the artists in working with such abstract forms of visual feedback (Fdili Alaoui 2012) ${ }^{2}$. Abstracted forms can be interpreted as having their own character traits when combined with sound or music and correlated in dynamics, intensity, and strength with the sound expression. Early precedents of this effect include the combination of animated abstract visuals and physicality (particles, boids, elastic models) seen in the pioneering work of Oskar Fischinger. Now with the ability to create a live synchronous synthesis of image and sound, novel artistic forms are being created. The major impact of such new frameworks is that a single physical model can be used to generate the dynamics of both image and sound in real-time, instead of tuning one medium to another (such as adapting animations to a musical score). Later in this article, we will present the new instruments of the art collective chdh such as emergence that generates both image and sound in real-time from a single physical model.

Mapping is a key component in real-time audio and graphic environments for the stage: it creates a relationship between inputs (movement) and outputs (graphical or sound synthesis programs). Basic mapping techniques are linear in that they associate a response that is proportional to the input. Such mapping techniques do not necessarily fit well within the expressive needs of the performance, instead, physical models can be used to control the mapping layer and make it more expressive (Momeni and Henry 2006). Another interesting feature of a physical mapper is its robustness to parameters' variations, which show that the tuning of such system is not restricted to a narrow range of parameter values and associated behaviours. The physical model plays the role of a real-time controller of parameters. Its behaviour gives a sense of physicality to the interactive relationship, enabling the performers to experience a more intuitive interaction with the physical model that is closer to the interaction with another live performer. An example of such physical mapping is illustrated later in this article with Effet Papillon that uses three 1D physical models to associate sensor inputs with parameters of sound synthesis.

\section{Laws and Computation of Mass Spring Systems}

Physical modelling generates simulated physical behaviours by simplifying the fundamental laws of

2 A set of experiments in collaboration with dancer and choreographer Marion Cavaillé have investigated the interest of physical models on stage, both for improvisation and for live performance. In the set-up, physical models were presented on a vertical screen, and the dancer was improvising in front of the screen watching the visuals and trying to improvise with them. Semi-directed interviews with the dancer have shown that she could 'play' with the models and progressively build up a choreographic relationship with the model as long as it could be perceived as responsive and at the same time endowed with autonomy (see section "Chiseling bodies" below). 
physics that govern these behaviours in reality. In particular, mass-spring systems (MSS) represent physical objects as masses and the interactions between them as springs/dampers. MSSs enable the modelling of clothes, muscles, or skin behaviours using a grid of masses and links with elastic properties corresponding to a simplification of the actual physical properties of the modelled material.

The ensemble of masses in a MSS interacts together in pairs through springs/dampers, and creates various dynamic behaviours. With the MSS, the links apply elastic forces on each mass following Hooke's law that states that the force needed to extend or compress a spring by some distance is proportional to that distance. Forces from the environment in which the masses operate, such as gravity, viscous forces, and other external forces, also govern the masses. Despite the simplicity of the law governing a single mass, when masses are connected to each other through an ensemble of springs, the numerous inner interactions can generate complex behaviours that result in complex dynamics. Technically, a MSS can be modelled as a graph in which the nodes are the masses and the links are the springs/dampers connecting pairs of masses, with three basic parameters: a rigidity value, a damping and a length at rest (or elongation). The set of values chosen for the physical parameters in a simulation, configures the set-up of the MSS and produces unique associated behaviours. Each set-up correspond to a different virtual object with its own rigidity, reactivity and inertia.

Newton's law is used to implement the motion of the masses and springs in space. This law describes the relationship between the acceleration of a mass and the sum of the forces applied to it $(\Sigma \mathrm{F}=\mathrm{m} \gamma)$. The relationship between acceleration and forces allows for the computation of a motion of a mass using discrete temporal steps by updating its position according to its acceleration and thus the forces applied to it. In other words, knowing the position of a mass at a given time step, the velocity and the sum of the forces applied to this mass, a time integration method allows us to compute the new position and velocity of the mass from one time step to the next (Georgii and Westermann 2005). Time steps have to be short enough to avoid errors due to interpolation that can result in a possible divergence of the model and the loss of its control. Thus, in order to implement a MSS into a computer program, it is necessary to perform two main arithmetic operations:

- one operation for summing the forces involved in Newton's law, i.e. the elastic and damping forces of the springs, and the external forces governing the masses,

- one operation for time integration and the computation of the mass motion.

On a computational level, a MSS can be implemented not only in 3D but also in multiple dimensions. A mass can be represented by a position vector in multi-dimensional space (over 3 dimensions vector). However, the most commonly used implementations restrain the mass movement in only 1 direction (respectively 2 or 3 directions) and obtain a 1D system (respectively 2D or 3D system). 
One of the simplest examples of a MSS consists of a single mass connected to a fixed point with a single

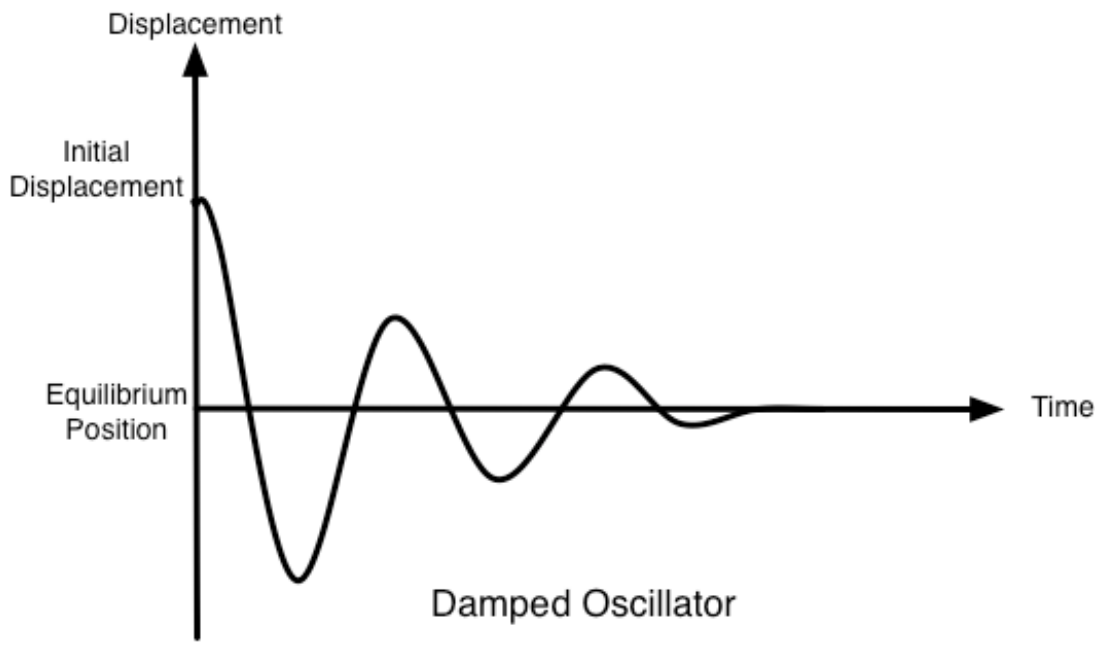

Figure 1: The mass position of a simple damped oscillator after having been displaced from its equilibrium position.

link, forced to move along a single axis, and governed by elastic and a viscous force. If the mass is displaced from its position of equilibrium, its dynamics are controlled by the sum of an elastic force proportional to the displacement (according to Hooke's law) and a viscous force with damping proportional to its velocity. When the mass is released, it oscillates with a fixed resonant frequency around the position of equilibrium and with decreasing amplitude over time. Such a system is called a simple damped oscillator and is illustrated in Figure 1.

Despite its simplicity, this system can be used in various ways. For example, when moving the fixed point, the mass position acts as a low pass resonant filter, while the spring elongation is a high pass filter. If we combine two simple damped oscillators, the system's oscillations are more complex and result in the interaction of the two frequencies of each single oscillator. Thus, a MSS combining dozens, hundreds, or thousands of damped oscillators composed of masses connected to each other, can generate very complex behaviours that can then be used to metaphorically evoke human movements or to control any digital process.

\section{Using MSS in Live Performance}

MSSs present various properties that make them attractive to use in the performing arts (Hsieh 2007; Jacquemin 2008; Johnston 2009; Fdili Alaoui 2012). They have the ability to produce physically plausible behaviours because they are governed by the laws of physics and hence have an evocative representation of real-world dynamics. We describe the behaviour of a physical model as physically plausible when it generates realistically perceived dynamics that are created autonomously to evoke real-world dynamics, without imitating real-world mechanics.

A MSS provides a wide range of controllable parameters in real-time (such as the parameters of the forces applied to the masses) that govern its behaviours, and thus offers rich possibilities of interaction. The MSS can be controlled through the mapping between the system's physical parameters and the features of the analysed movement. The resulting interaction creates a relationship between the performer's 
movement and the associated MSS behaviours when following pre-defined rules or interaction scenarios.

MSSs simulations generate data streams that can be used to control different media. The applications described in this article are limited to sound and visual media, but other actuators such as lighting devices can also be controlled using a MSS where the data generated, can be directly used as a synthesis kernel. The 'scanned synthesis' method by Verplanck et al. (2000) for example, uses a MSS to generate shapes, which build waveforms for audio synthesis. The use of MSSs for a parallel control of different media can ensure a strong recognisable coherence between these media. The same physical simulation can generate data concurrently synthesized into sound and graphics. In real life a moving object's velocity and acceleration produce sound while the viewer perceives the object's position in time. Thus to generate plausible cross-media behaviours, one can simulate real life cross-modal phenomenon and use the mass positions to control the graphical rendering and their velocities and accelerations to control the sound synthesis. The parallel multimedia control allows us to perceive the audio-visual phenomenon with more consistency as both sound and visual behaviours are produced from the same physical simulation.

\section{Four Case Studies}

In this article we are focussing on four applications where we consider the use of physical models as a digital partner to a real-life performer. This can only be achieved through a careful design of the model and a genuine hybrid coherent choreography both for the performer and the MSS. These applications illustrate various ways to use MSSs for the design of a performance and are not meant to be exhaustive but rather illustrative. Indeed there are as many ways to use MSSs in performance as the plethora of possible correspondences that can be established between the physical model and its real-world alter ego the performer.

In Effet Papillon the use of a MSS illustrates a variety of options for performers to manipulate sound based on their choice of movement. This particular application shows the potential of artistic manipulation of sound when it responds to captured physical movement. The dance installation Double Skin/Double Mind shows how a MSS can be used as a metaphorical, physically plausible echo of dance movement qualities. Here we illustrate how dancers can benefit from shifting their perception of movement when interacting with a physical 'double' in a pedagogical context. Chiselling Bodies reflects upon the notion of a digital alter ego in a dance performance, by creating an improvisation space fostering creativity between live and digital performers. The final case study chdh is an audio-visual performance that immerses the audience into a multi-modal performance in which the dynamics of sound and live graphics are perceived as part of the same physical process modelled using a MSS.

\section{Effet papillon}

Effet papillon ${ }^{3}$ (Butterfly Effect, 2007) is a dance performance created by the French dance company Contour Progressif (Figure 2) and explores ways of providing digital sonic representation of the human body. Three dancers reproduce the movements of a digital avatar while being immersed into a virtual environment with interactive sound and lighting. The virtual environment reacts to the dancer's movements to increase the sensation of the body when adjusting to a virtual performance space. Cyrille

3 Documentation of Effet papillon performance of Contour Progressif in collaboration with Cyrille Henry http://www.contour-progressif.net/projets/spectacle/effet-papillon 
Henry developed the capture and analysis engine of the movement data and contributed to the artistic

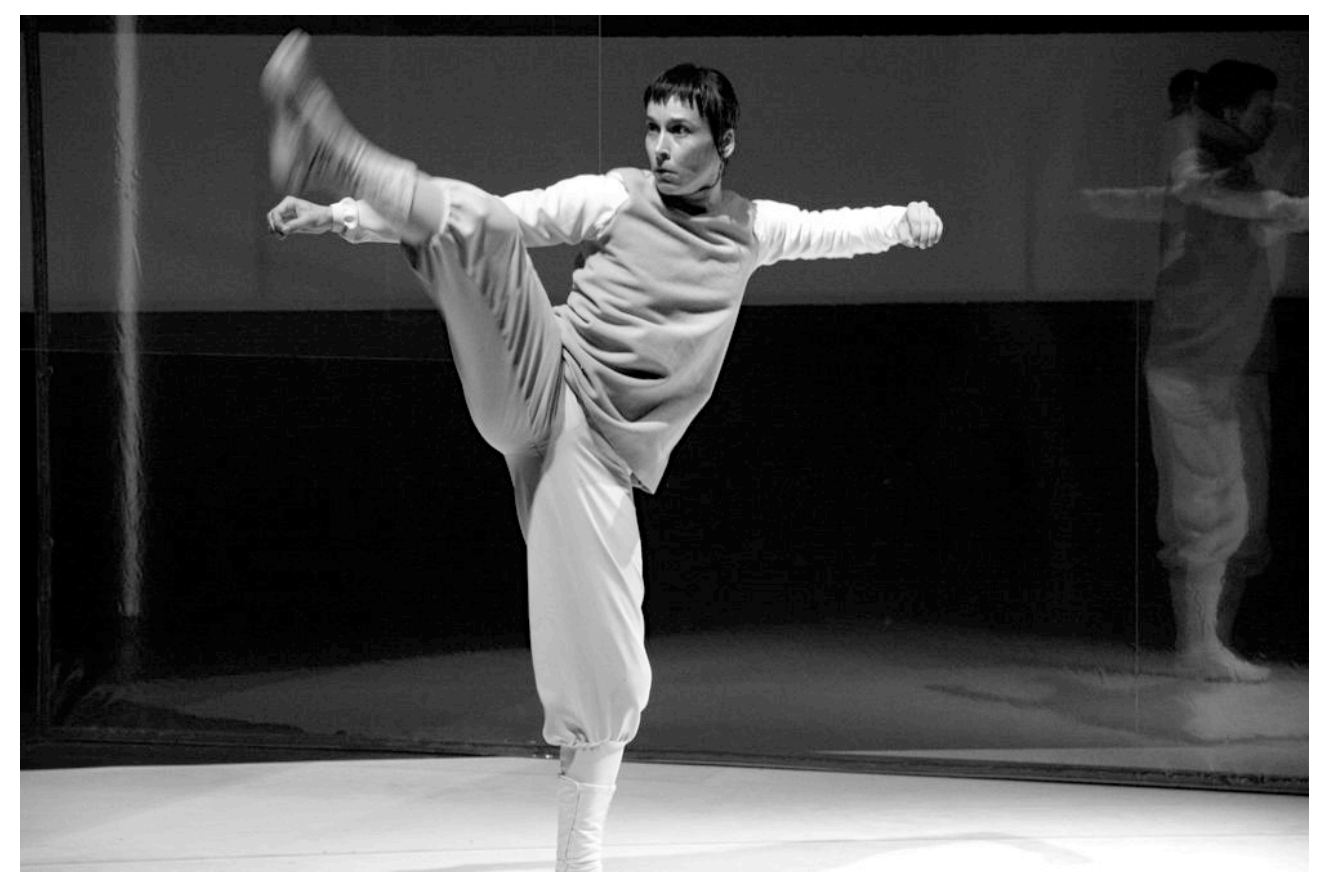

Figure 2: Effet papillon performance (butterfly effect) in collaboration with Contour Progressif Dance Company. (C) Mathieu Bouvier/Contour progressif

process of Effet Papillon.

Various sensors are used to capture each of the three dancers' movements. Bend sensors are placed on the knees or the elbows and accelerometers are located on the wrists or the ankles. The real-time data capture is sent through a wireless network to control the audio synthesis. This data is filtered using MSSs acting as simple damped oscillators. The capture data from the wearable sensors are then able to control the audio synthesis. The MSS is used to create sound and lighting that both have their own unique behaviour, and at the same time are controlled by the dancers' movements.

The MSS damped oscillator filters have different goals:

1. Richness and diversity of the sound (From 1D to 3D): We used three 1D MSSs made of a single MSS as resonant filters, controlled by the performer's acceleration amplitude. We chose different pre-sets of physical parameters for each of the three filters to create a bouncing behaviour. The three resulting MSS outputs are thus distinct even if they all move according to the same input. These different outputs are then used to control different parameters of the audio synthesis. Such a dynamic mapping allows the audio synthesis to evolve in a 3D parameter space, rather than a $1 \mathrm{D}$ space. It allows for more diversity and richness of the produced sound.

2. Coherence (Expressive sound produced by MSS behaviours): Each 1D MSS has its own resonant frequency and this produces identifiable behaviours. Although the three MSS behaviours vary according to their physical parameters, they are perceived as a single body that helps to identify a distinctive sound source. 
3. Augmenting movement: When directly mapping a movement to a sound synthesis, the produced sound acts as a mirror of the movement. Adding a MSS layer to filter the captured movement and map the movement to the sound synthesis, produces sound that is not a direct representation of the movement anymore but rather a representation of an invisible object that is related to the movement. The use of physical model simulations to filter the captured dance movement has been strongly motivated by artistic choices: it aims at creating a 'distance' between the dancer's movement and the produced sound in order to avoid mirroring the movement that is already visible. Thus the produced sound is meant to augment the perception of the movement by being indirectly manipulated by the dancer.

\section{Double Skin/Double Mind}

Double Skin / Double Mind is a dance training workshop given by the company Emio Greco | PC. Since 1996, it has served as the basis of the company's dance vocabulary. The workshop incorporates lengthy breathing exercises focussed on expanding the body (Breathing component), rebound, jumps and changes of rhythm (Jumping component), extreme expansions (Expanding component) and freezing moments (Reducing component). These intensive movement sequences are used to provide an awareness of the dancer's inner body. (Bermudez and Fernandez 2010).

Merging daily practice with the artistic and ideological urge to discover new means for transmission, the company initiated a research project using interactive sound and visuals as ways to transmit its vocabulary in an interactive installation, Double Skin Double Mind $(D S / D M)^{4}$ (see Figure 3).

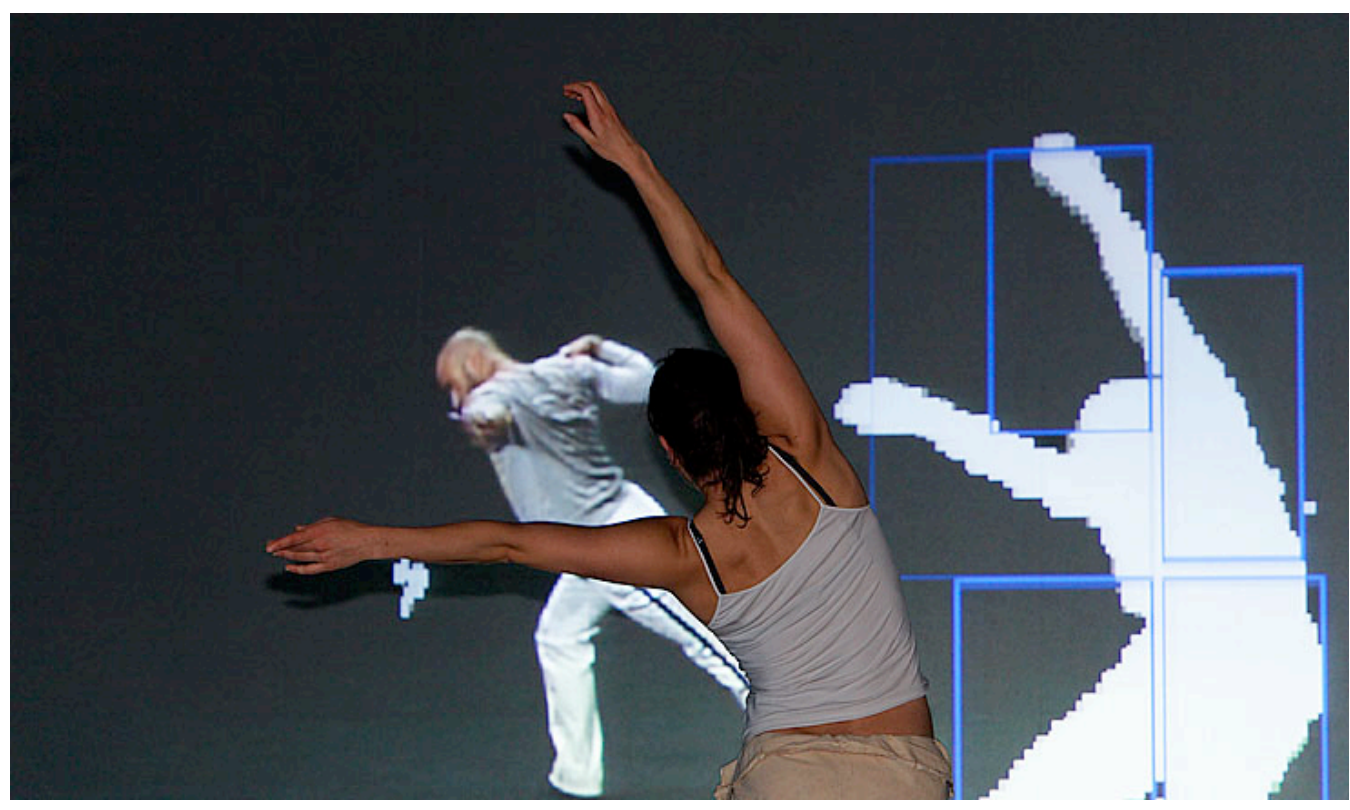

Figure 3: Double Skin/Double Mind pedagogical interactive installation for Dance. CThomas Lenden

4 Videos of DS/DM installation: http://www.researchcatalogue.net/view/?weave=1585 and http://sarah.alaoui.free.fr/spip.php?article1 
Sarah Fdili Alaoui collaborated with Emio Greco | PC to design the interactive installation DS/DM with a specific focus on using interactive visuals as a new way to teach the company's dance movement qualities and to increase the dancer's inner body awareness. The installation produces an interactive visual feedback based on a simple MSS, controlled by the dancer's movement qualities when undertaking the core workshop components of Breathing, Jumping, Expanding, and Reducing. In DS/DM the main focus is on the notion of 'movement qualities'. In contemporary dance, 'movement qualities' define the qualitative attributes of movement with respect to one's inner attitude or intention. Movement qualities essentially convey information revealing movement's expressiveness. For example, the theory of Laban Movement Analysis (developed by Rudolf Laban) describes movement expression as Efforts qualities that can be experienced and observed as an attitude shift revealing the mover's intent in response to the environment (Laban, 1947).

In an early user study of $D S / D M$, the dancers reported that interacting with the visuals allowed them to perfect their performance of the workshop components and to explore and generate new movement material (Fdili Alaoui 2013a). This feedback mechanism is achieved through the ability of the system to recognise the performed movement qualities in real-time and to respond to them in turn through the evocative behaviour of the MSS. The final goal is to teach the dance material but also to give the learned material a life of its own so that the dancer can feel free to develop his/her practice while interacting with the visuals as a digital performer.

The design of $D S / D M$ interactive visuals is an aspect of Sarah Fdili Alaoui's research in which she is focusing on movement expressivity in interactive systems for dance through the use of movement qualities as an interaction modality. In the context of $D S / D M$, her approach is to design the visual behaviours of the MSS to create expressive experiences for the dancer through both the real-time analysis and representation of the dance movement qualities. Sarah Fdili Alaoui collaborated with Bertha Bermudez from the company, to develop a small scale MSS with physically plausible behaviours (right image of the Figure 4) and a mapping strategy to control it (shown in Table 1). The mapping associates the dancer's extracted movement features with the physical parameters of the MSS responsible for the generation of different types of physical behaviour. Each range of movement quality features can control different MSS behaviours, e.g. a mass rebounding or a mass evolving in a viscous environment, that metaphorically responds to the dancer's movement qualities in real-time.

\begin{tabular}{|l|l|l|}
\hline $\begin{array}{l}\text { Double Skin/Double } \\
\text { Mind movement } \\
\text { Component }\end{array}$ & Movement Qualities features & MSS parameters \\
\hline \multirow{2}{*}{ Breathing } & Verticality & Stiffness of visible springs \\
\cline { 2 - 3 } & Quantity of motion & Environment viscosity \\
\hline \multirow{2}{*}{ Jumping } & Periodicity & Stiffness of visible springs \\
\cline { 2 - 3 } & Quantity of motion extension & Rest length of visible springs \\
\hline \multirow{3}{*}{ Expanding } & Quantity of motion & Stiffness of visible springs \\
\cline { 2 - 3 } & Legs opening & Rest length of visible springs \\
\cline { 2 - 3 } & Quantity of motion & Environment viscosity \\
\hline \multirow{2}{*}{ Reducing } & Quantity of motion & Rest length of visible springs \\
\cline { 2 - 3 } & Shifting of weight & Environment viscosity \\
\hline
\end{tabular}


Table 1: The mapping between the movement qualities parameters and the MSS parameters during the active state of the four visible masses and springs.

The interactive system for DS/DM are described in detail in (Fdili Alaoui et al. 2013a) and relies on a movement capture and a recognition layer that links the performed movement with pre-recorded and tagged movement qualities of Breathing, Jumping, Expanding, and Reducing in real-time. The MSS is composed of four visible masses connected with each other through four visible links initially in a square shape (left image of the Figure 4). The MSS is also composed of four invisible fixed masses connected to the four visible ones through invisible links. These invisible masses are used to bring back the visible masses to a square shape when the dancer stays still by deactivating the forces of the visible links and activating the forces of the invisible ones. When the dancer's movement is recognized as a DS/DM component, the system returns to an active state in which the four visible masses and links adapt their behaviour to the identified movement qualities. We chose to work with a small scale MSS that enables a precise control of each mass based on the extracted movement qualities.

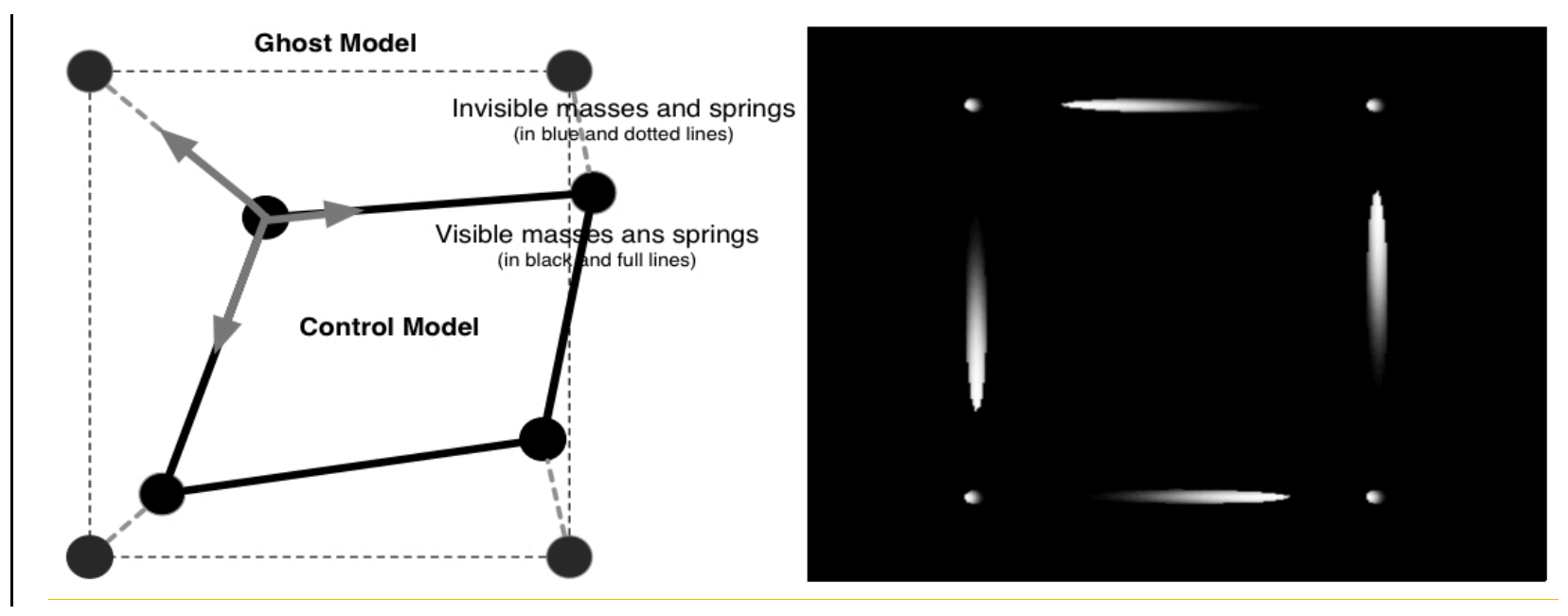

Figure 4: The MSS topology used and visual rendering in Double Skin/Double Mind

By displaying visual feedback that represents the user's movement qualities, our MSS aims at helping the dancers to improve their execution of Emio Greco | PC qualities. This is a new approach for dance pedagogy where new media is meant to enhance the learning process of dancers. For this reason, the installation has been welcomed by dance pedagogy professionals and used every year as a pedagogical tool in the dance curriculum of the department of the Amsterdam School of the Arts.

\section{Chiselling Bodies}


The Chiselling Bodies 5 augmented performance was initiated and developed by Sarah Fdili Alaoui in collaboration with Marion Cavaillé, a ballet dancer at the Ballet National of Marseille. The concept of the performance is that the dancer interacts with visuals to create an improvisational duet between her dance movement and the visuals' abstract dynamics (Figure 5). Both Sarah and Marion jointly choreographed a structured dance improvisation and a narrative for the interactive visuals to create an echo between the movement and the virtual behaviour without precisely revealing the model response to the movement. To design a visual metaphorical echo of dance movement, a massive MSS is used (right image of Figure 6). Its dynamics respond to the movement qualities extracted in real time from the dancer's performance as shown by Fdili Alaoui et al. (2013b).

The system is composed of a motion capture layer based on wireless accelerometers combined with the infrared sensor Microsoft-Kinect as well as an image processing program to reconstruct a skeleton from the silhouette of the dancer. The skeleton is used to acquire the $3 \mathrm{~d}$ positions of the dancer's head, hands, center of mass, and feet.

The system extracts the following dancer's movement features from the motion capture data:

- the kick (gestures impact) index,

- the hand energy,

- the jump/drop index,

- the verticality/height, and

- the stillness index.

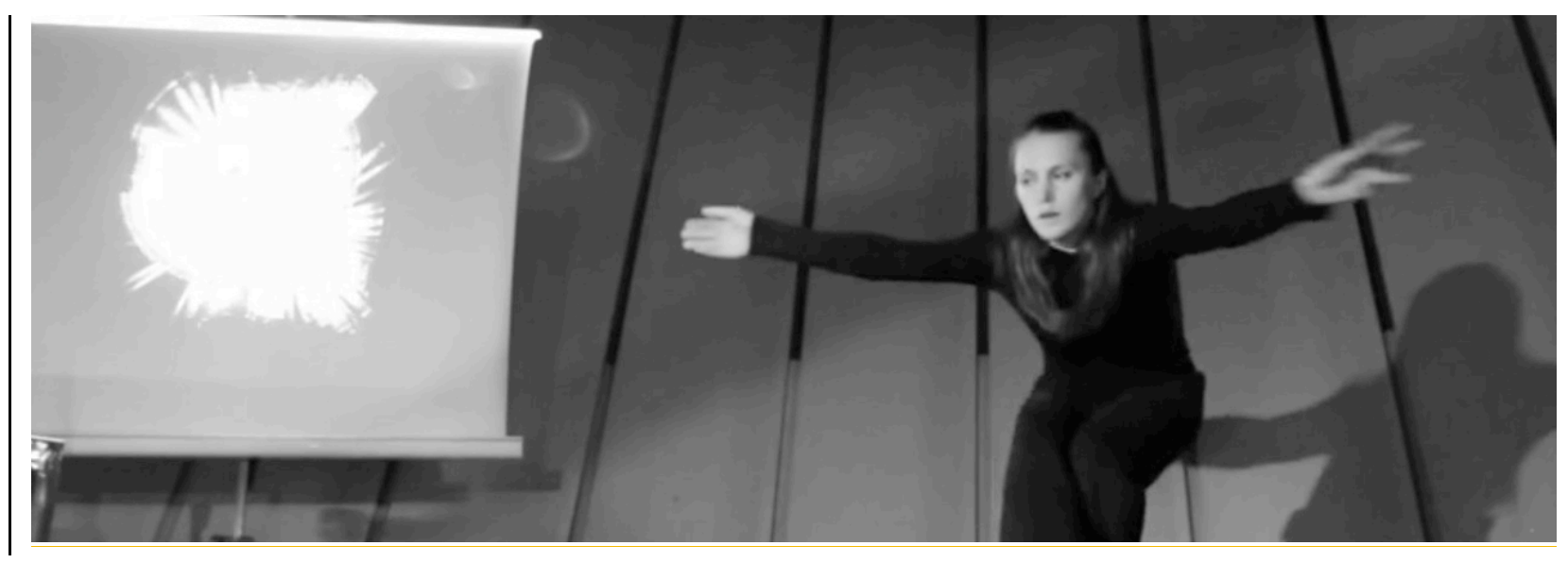

Figure 5: Chiselling Bodies Augmented Dance performance.

In order to generate in real time visual behaviours metaphorically responding to the dancer's movement

5 Video of the rehearsal of the performance Chiseling Bodies:
http://sarah.alaoui.free.fr/spip.php?article34 
qualities, Sarah Fdili Alaoui developed a massive MSS (large number of masses and links, up to 250,000) with a hierarchical topology (left image of Figure 6). Each of the four heavy visible masses are linked by elastic springs to over 60,000 light visible enslaved masses arranged in a circle around it. Four ghost invisible masses are linked to the four heavy ones in order to stabilize the MSS whenever the dancer is still.

Sarah Fdili Alaoui used a participatory methodology involving the dancer to design a mapping layer linking the MSS parameters with the extracted features of movement. This mapping is designed to tune the MSS visual dynamical behaviours according to the dancer's movements following the commonly defined interaction scenarios summarized in Table 2. The participatory design methodology consists of cycling through the steps defined by Spinuzzi (2005) and Coughlan and Johnson (2009):

- brainstorming to produce and sketch ideas, metaphors, and inspirations for the performance and to define interactive scenarios (Table 2),

- prototyping and testing of the interactive system,

- reporting and questioning each other's observations of the testing phase.

Such a methodology is driven by a common desire of joint collaboration between the scientific researcher Sarah Fdili Alaoui and the dancer on both the choreographic and computational elements of the performance. The resulting system merges the technology with the choreography enabling the dancer to acquire a better understanding of the behavioural and dynamic possibilities of the technology e.g. the physical model, and even further explore its constraints in creative ways.

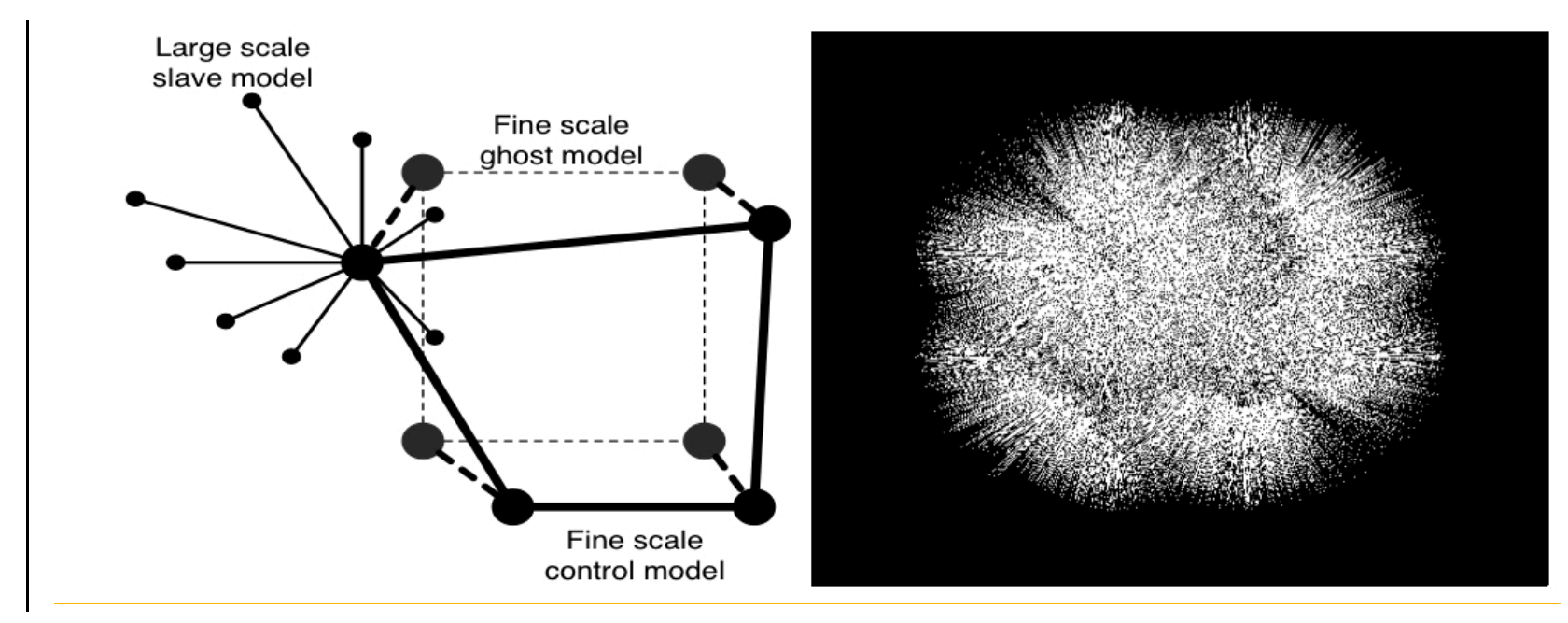

Figure 6: The MSS topology used and visual rendering in Chiseling Bodies 


\begin{tabular}{|l|l|}
\hline Chiselling Bodies movement behaviours & Expected MSS visual behaviours \\
\hline Slow energy of the hand & Symmetrical rosettes forms \\
\hline Continuous energy of the hand & Blossoming effect \\
\hline Foot kick & Chaotic effect \\
\hline Drop/jump & Gravity effect \\
\hline Verticality/height & Weightlessness effect \\
\hline Transition stillness/movement & Solidified/smooth effect \\
\hline Transition between movements & Solidified/smooth effect \\
\hline
\end{tabular}

Table 2: The Interaction scenarios linking the movement behaviours with the MSS visual behaviours.

At the end of the participatory design cycles, Sarah Fdili Alaoui interviewed the dancer about her experience of the final prototype of the interactive system. The interview was recorded and a qualitative analysis of the transcriptions of the dancer's feedback was used to assess her experience of the interactive installation.

The results of the interviews showed that the dancer perceived the visual feedback as an echo and a 'transcription' of her movement. She perceived the MSS dynamics as related to the energy of her movements. This is consistent with the mapping used to link the energy of the hand or the foot movement with various visual effect such as a 'rosette', a 'blossoming' or a 'chaotic' metaphoric visual effect. These visual effects evoked different forms that the dancer imagined to be painting with her movements. The dancer also reported on the capacities of the visuals to have self-agency by varying their response and creating an effect of surprise. The dancer described the visuals as being alive just like a dance 'partner' creating a duet with her. Finally, the dancer's overall experience of interacting with the visuals based on massive MSS was positive, as she qualified them by 'beautiful'.

Drawing on the interview responses, we can propose that the MSS creates an improvisational tool with two main values:

1. Metaphor and dialogue: The dancer's movements control the MSS so that its dynamic behaviours illustrate a direct but independent connection, which evokes, reflects and responds to the dancer's movements. This metaphorical relationship drives the dancer towards a 'dialogue' between her movement and the semi-autonomous behaviour of the virtual model.

2. Generation of unpredictable dance forms: Although the visuals do not represent a human silhouette, their physicality drives the viewer and the dancer to accept their patterns as dance forms. Moreover, due to the massive scale and topology of the MSS, the system is capable of generating a wide range of unpredictable semi-autonomous behaviours (and thus dance forms). By creating endless possibilities of dance forms on stage. Thus, the system can be seen as a digital dance partner.

\section{chdh}

$\mathrm{chdh}^{6}$ is an artist collective currently composed of Cyrille Henry and Nicolas Montgermont, who have been creating audiovisual performances together since 2002. chdh's body of work is based on the expressiveness of the movement of sound and images. Both sound and images are selected to be

Artist collective chdh: www.chdh.net 
minimalist and abstract in order to emphasize their dynamic behavioural patterns without literally representing a recognizable form such as a human silhouette. The sounds and images are closely related to each other in that both media are abstract representations of the same behaviours, resulting in a direct, but not redundant, connection between the media. The chdh collective generate live synchronous sound and images in real-time using a single physical model that provides a high cross-media consistency, instead of tuning one medium to another such as tuning the visual according to the sound or vice versa. During performances, both artists are controlling a MSS that drives both the sound and image media indirectly. chdh considers the combination of the MSS, the sound and images and the performer interaction with the MSS, as a sophisticated tool for performance that they call an 'audiovisual instrument" . We will explore two of these instruments "Latent" and "Emergence" in order to illustrate how the MSS permits the real-time connection between the sound and the image.

\section{"Latent":}

chdh chose to design a complex MSS for "Latent" with the aim to:

1. create an audio-visual instrument that is easy to perform using few control parameters,

2. achieve a cross-media consistency,

3. create rich and complex audio-visual behaviours,

4. create an audio-visual instrument generating predictable behaviours.

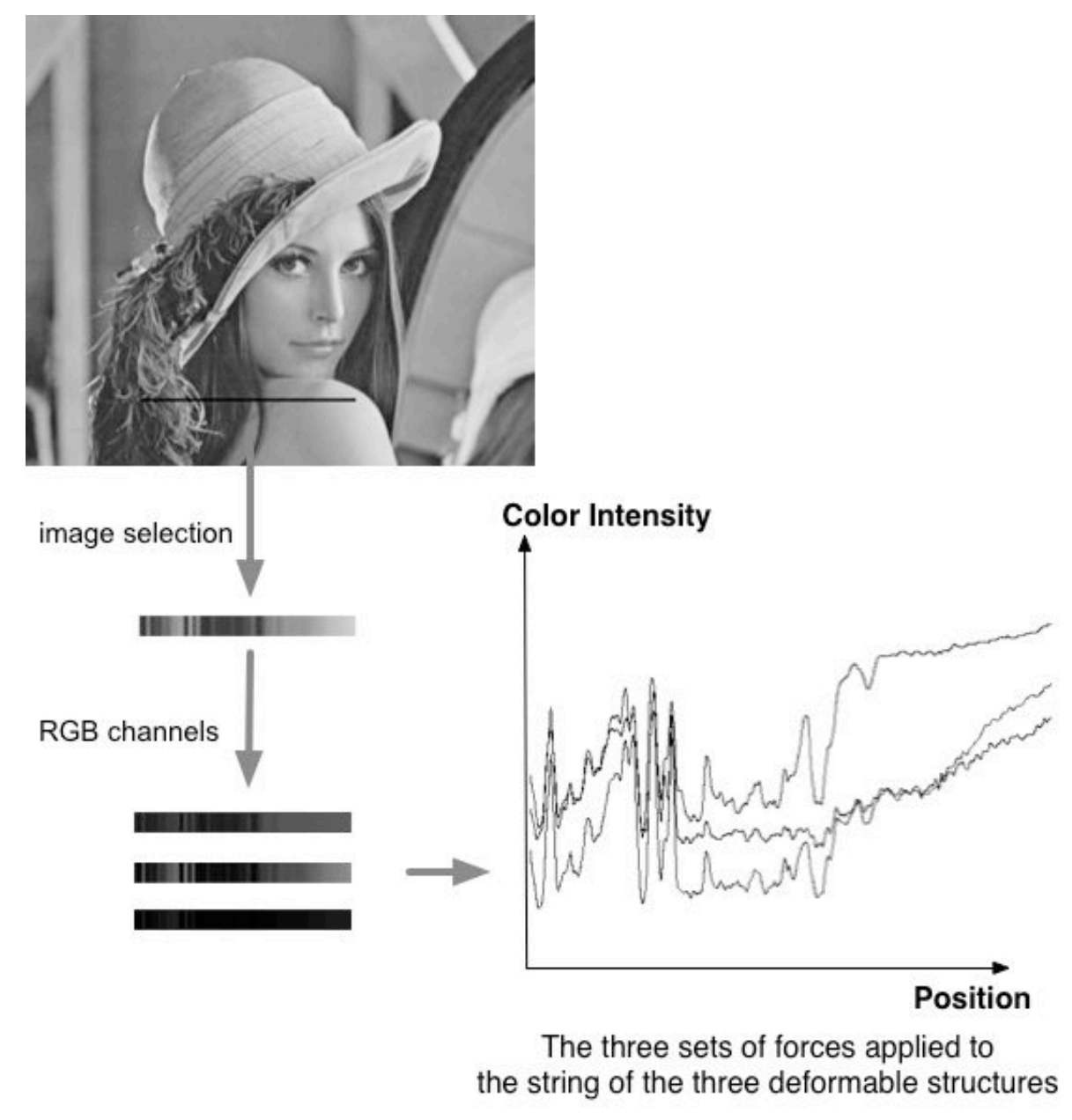

Figure 7: image, selection and resulting RGB channels used for the structures shapes. 
To fulfil these goals, "Latent" was built upon three identical 1D MSSs with a string topology: masses are connected by springs to each other to form a complete circle. The three MSSs are connected to three deformable virtual structures that can be distorted in order to disturb the string equilibrium. The masses in each string are connected to the masses of the corresponding deformable structure. These three structures can be distorted by the user's input, in three different shapes. The most crucial and challenging part for the design of this instrument is to find a way to control the different shapes of the deformable structures. Since these shapes are used for both the sound and the visual synthesis, their complexity impacts directly on the complexity of the sounds and visuals that are produced. To generate these shapes, chdh uses prerecorded data from the three RGB channels of various digital pictures (see Figure 7). In each image, a region is chosen in which each pixel channel controls a mass of one of the three structures. By moving the pixels region in both directions in the image continuously the colour data changes and thus the shapes of the three structures are also altered.

"Latent" provides some control parameters, firstly via the physical properties of the spring links (elastic and the damping values of the springs), and secondly, through the shape of the deformable structures. The sound produced by this instrument is based on a scan synthesis: the shapes of the three strings are used as the basis of sound wave shapes ${ }^{7}$. The graphical representation of this instrument relies on a series of rectangles arranged in a circle (Figure 8). The shape of the three strings controls the scale and the translation the rotation angle of each rectangle. The physical models are interpolated to represent a continuous system, so that the number of rectangles does not necessarily fit the number of masses in the

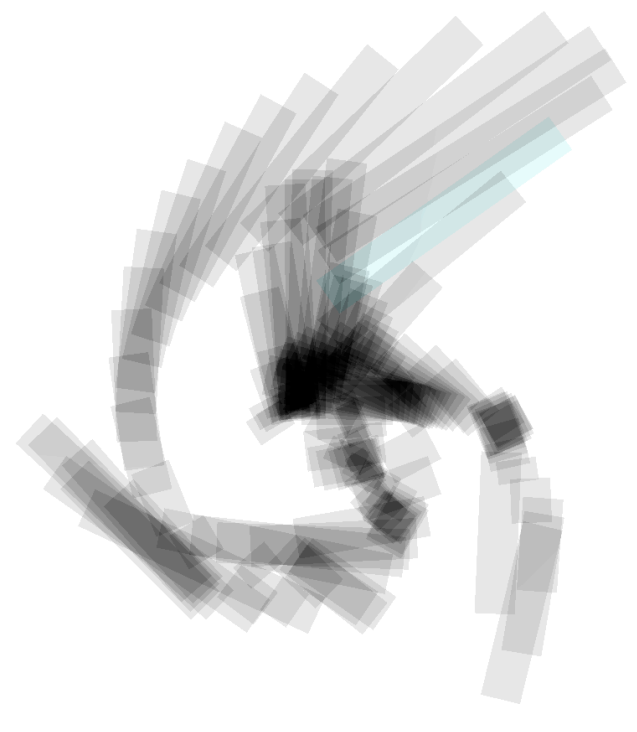

Figure 8: "latent" instrument visual representation.

MSS string. 
The image used to control the shape of the string can be changed to obtain different structures and different correlations with the corresponding pixel channels. Using an image that is very smooth will result in bass sounds with low harmonics and uniform transformation of the visual rectangle. Using a very granular image will result in high frequency harmonics and more chaotic rectangle alignment. Using a black and white image will allow less diversity than a colourful image in both sound and visuals. In this application, using a MSS allows the reduction or enhancement of the harmonic structure of the initial shape and its possible variations. Here the MSS creates a dynamic mapping that is used to creation a rich and usable instrument.

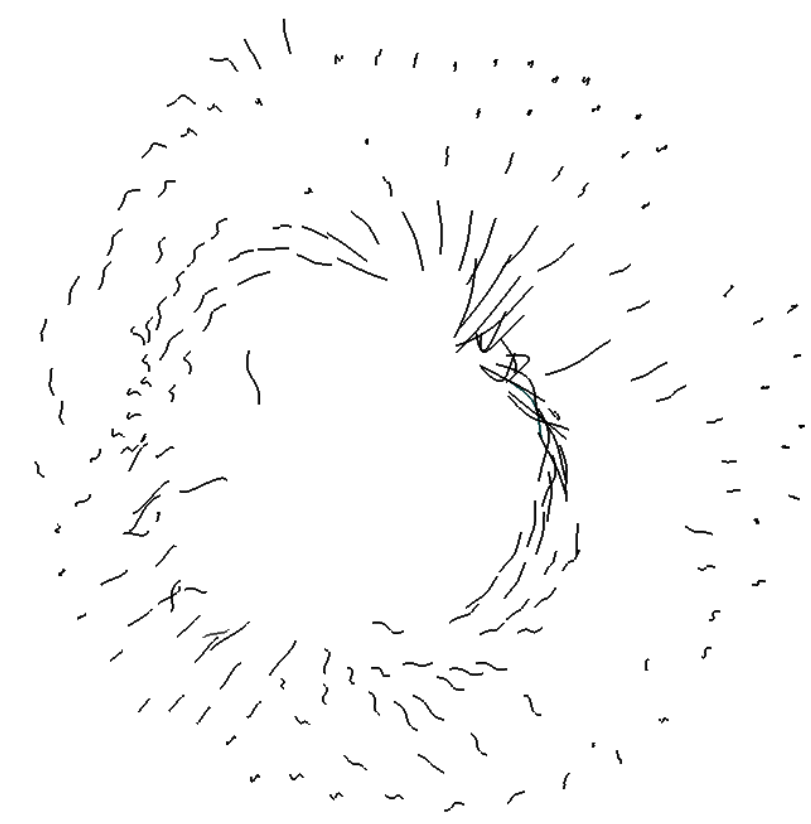

Figure 9: emergence of spatial arrangements in visual instrument 


\section{"Emergence"}

"Emergence" was used to produce the audiovisual art pieces that constitute the album vivariu ${ }^{m 8}$ as well as the audiovisual pieces of the performance egregor ${ }^{e 9}$. This instrument relies on a simple MSS, but produces complex behaviours. The model is based on approximately two hundred identical masses that are connected to each other via a non-linear connection point. Depending on the shape of the function curve that is used to compute the forces associated with each spring elongations, the masses organize themselves in different arrangements. While all the masses and links are identical, and no additional external force is applied to the masses, various spatial arrangement and rhythmical patterns can still emerge (Figure 9). It is worth noting that if the forces are too strong the masses will not stabilize in a point of equilibrium, but will keep behaving in a spatially organized way, hence generating different kinds of movement and visual arrangements.
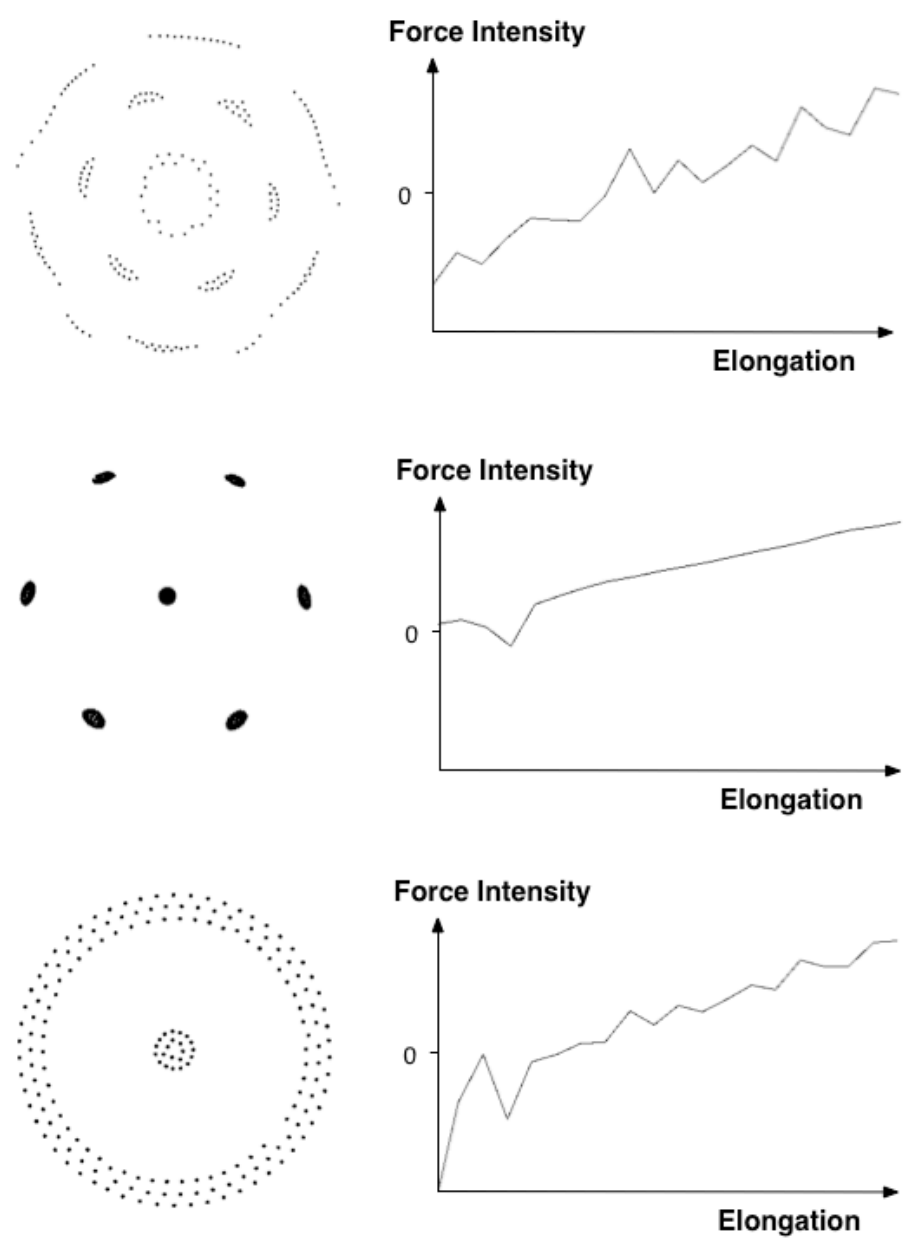

Three static spatial arrangement

Three corresponding forces

Figure 10: Different static spatial arrangements and the corresponding function curves used to compute the spring forces from their elongations. 
Different function curves are used to associate the spring forces with their elongation. The control of all the masses' behaviours is made by interpolating between such predefined functions. Since the behaviours of the masses are very complex, we use a straightforward visual representation. Each mass is used to produce one sound and to visualise one curve described through the last mass positions (Figure 10). For each mass, an audio oscillator is used, modulated by a filter. The velocity of the mass controls the filter frequency as well as the sound amplitude and the sound of the instrument results from the addition of all the sounds produced by all the masses.
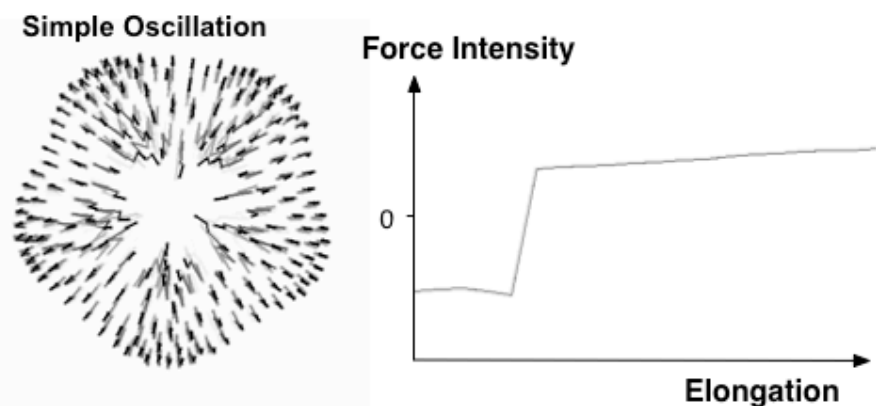

Average radial velocity
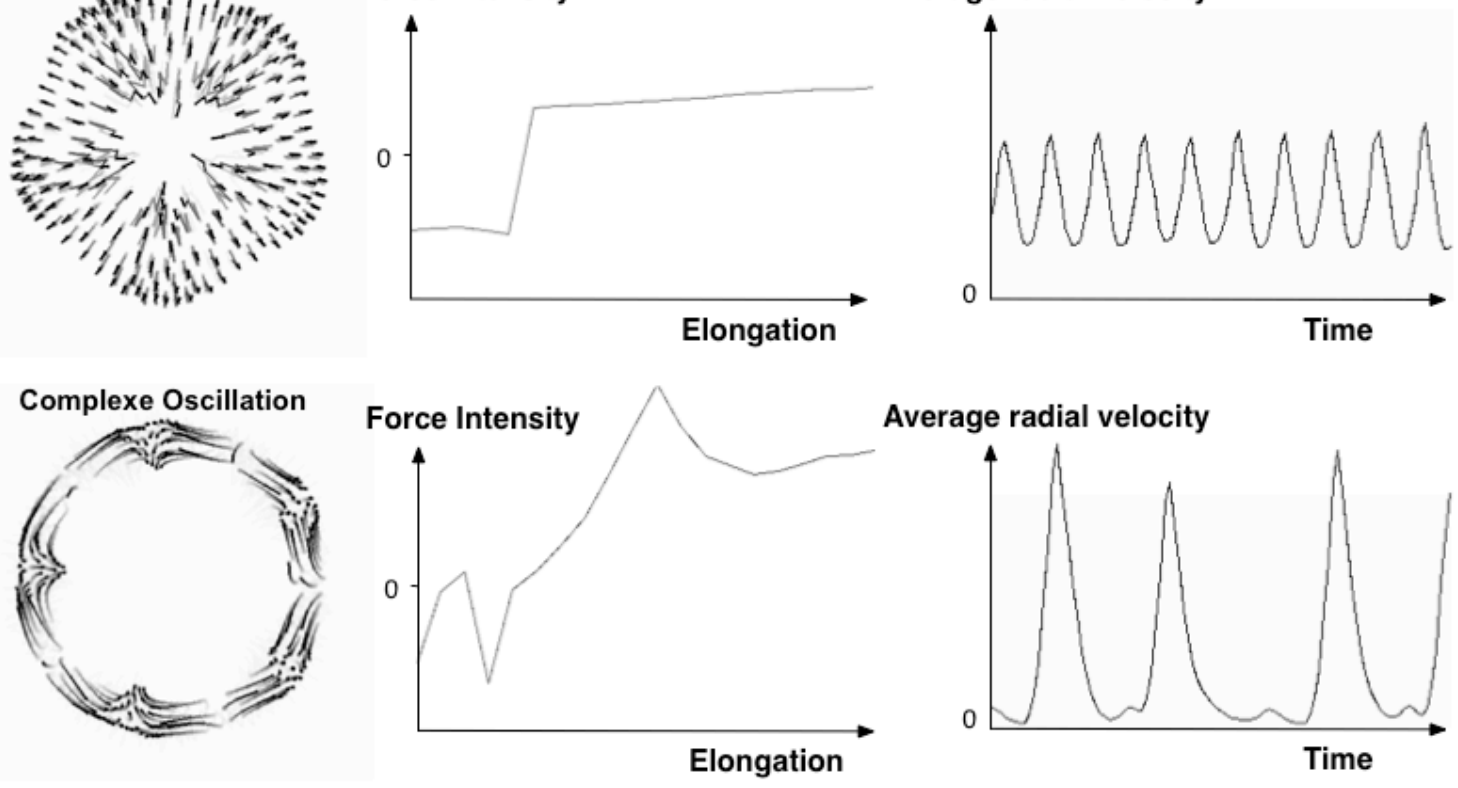

Average radial velocity

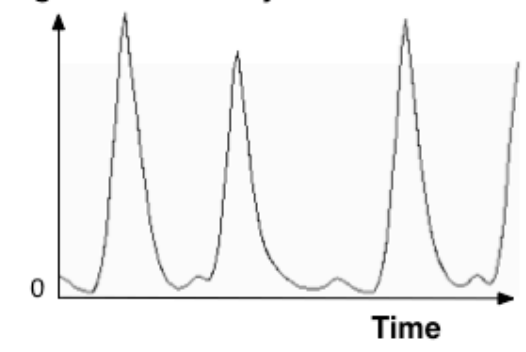

Chaotic Oscillation

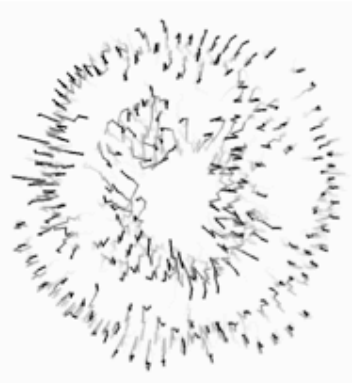

Force Intensity

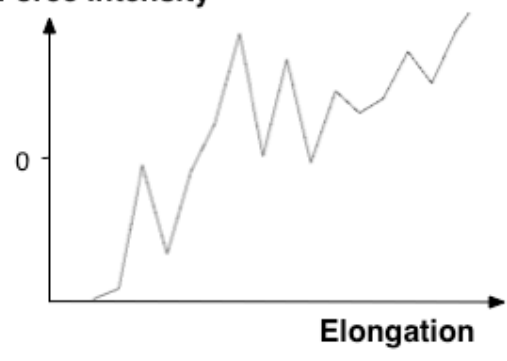

Three corresponding forces
Average radial velocity

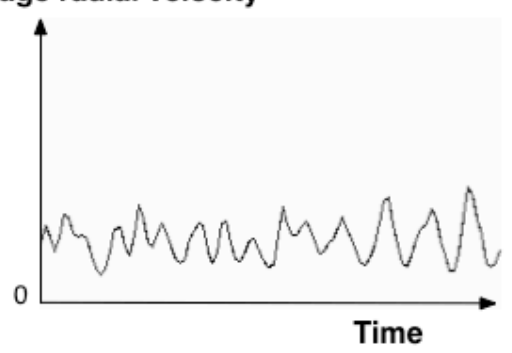

Average radial velocity of the masses

Figure 11: rhythmic patterns i.e. spatial mass configurations, function curves used to compute the spring force as a function of its elongation, and the mass average radial velocities over time in three different cases: simple oscillation, complex, and chaotic rhythms. 
Thanks to the use of a MSS, this instrument offers a very large range of complex behaviours, from highly organised structures to almost chaotic movements (Figure 11). Despite its multiple behaviours, the MSS can still be controlled in an accurate way because of the design choices used to select the available control parameters, allowing for its ease of use in real-time performance contexts.

In emergence, as well as in most chdh audiovisual instruments, sound and images are created using two different sets of data coming from the same MSS. In this work, the sound is never a direct representation of the images, nor the image of the sound. Nevertheless, they share a common source of control. Since both media represent the same abstract behaviours, the audience perceives a strong connection between them. In feedback from chdh audiences, the instruments have been described as having plausible physical behaviours sometimes using analogies with animals or natural phenomena.

\section{Conclusion}

Through these four art-science collaborations with dancers and researchers in digital media, we have illustrated the contribution of physical models as digital partners for performers. The applications can be used not only in live performance, but also dance pedagogy, digital instrument design and improvisation. It is clear from our experience that the model set-ups (topology, parameters, mappings) play a crucial role as a MSS does not develop a single and formatted behaviour. The designer's skill lies in tuning a model, adapting its behaviour to each performance and designing the controls that will be used for real-time interactions in live performances. The performers' skill lies in their capabilities to develop partnerships with the digital companion, reflect on the embodied and perceived values of the interaction with the resulting behaviours and provide feedback for further opportunities to creatively exploit the physical models for supporting their artistic expression.

While MSSs are one of the most commonly used physical models in Digital Art for modelling and simulating 'natural' physical processes, particles and fluid dynamics, L-systems, diffusion and percolation processes, and crystals are among the many other physical models that could be further explored as digital partners for the stage. These physical models would certainly offer new perspectives on the applications of digital media for performance due to their specific physical properties that distinguish them from each other.

Another artistic perspective and future research direction concerns physical modelling for movement metaphors. MSSs have been presented in a fairly low-level technical way with details about forces, damping, and topologies, they can be considered as an interface for which the major issue is not the technical programming of the physicality, but a deeper reflection on the expected behaviour of the model in response to the various user input scenarios. For this purpose, the interaction designer should not focus on mappings between a user's movement analysis and parameter values of the models, but rather on higher-level notions such as affective feedback or user experience. This article offers a step in this direction by highlighting typically ignored features of the interactive movement qualities that have connections with the emotions of the user and her corresponding expression. Through such concepts as movement qualities we wish to pull interaction design of creative physically inspired interfaces towards more empathic and sympathetic behaviours to better foster expression and creativity. 


\section{References}

Bermudez, Bertha and Carla Fernandes. 2010. "Inventing the interactive glossary: an approach to documenting contemporary dance.” Arti journal 2 (2): 29.

Coughlan, Tim and Peter Johnson. 2009. "Understanding productive, structural and longitudinal interactions in the design of tools for creative activities." in Proceedings of the seventh ACM conference on Creativity and cognition. ACM, New York, NY, USA, 155-164. DOI $=10.1145 / 1640233.1640258$

Coros, Stelian, Andrej Karpathy, Ben Jones, Lionel Reveret, and Michiel van de Panne. 2011. "Locomotion skills for simulated quadrupeds." ACM Transactions on Graphics 30 (4): 12 pages. DOI $=10.1145 / 2010324.1964954$

Demoucron, Matthias. 2008. "On the control of virtual violins - Physical modelling and control of bowed string instrument.” PhD diss., UPMC, Paris, France.

Dixon, Steve. 2007. Digital Performance: A History of New Media in Theatre, Dance, Performance Art, and Installation. Cambridge, MA and London: The MIT Press.

Fdili Alaoui, Sarah. 2012. "Dance Gesture Analysis and Visual Feedback based on Physical Models: Contributions of Movement Qualities in Whole Body Interaction." PhD diss., Univ Paris Sud, Orsay, France.

Fdili Alaoui, Sarah, Frederic Bevilacqua, Bertha Bermudez, and Christian Jacquemin. 2013a. "Dance interaction with physical model visualization based on movement qualities." International Journal of Arts and Technology 6 (4): 357-387. DOI=10.1504/IJART.2013.058284

Fdili Alaoui, Sarah, Christian Jacquemin, and Frédéric Bevilacqua. 2013b. "Chiseling bodies: an augmented dance performance." in CHI '13 Extended Abstracts on Human Factors in Computing Systems. ACM, New York, NY, USA, 2915-2918. DOI=10.1145/2468356.2479573

Georgii, Joachim and Rüdiger Westermann. 2005. "Mass-spring systems on the GPU." Simulation Modelling Practice and Theory 13 (8): 693-702.

Gregory, Richard L. 1970. The intelligent eye. New York: McGraw-Hill.

Hsieh, Chi-Min and Annie Luciani. 2005. "Generating dance verbs and assisting computer choreography." in Proceedings of the 13th annual ACM international conference on Multimedia. ACM, New York, NY, USA, 774-782. DOI=10.1145/1101149.1101314

Hsieh, Chi-Min. 2007. Grammar of Physically Based Modeling of Dance Movements: Use for Choreographic Composition. PhD diss, INP Grenoble, France.

Jacquemin, Christian. 2008. "Allegra: a new instrument for bringing interactive graphics to life." in Proceedings of the 16th ACM international conference on Multimedia. ACM, New York, NY, USA, 961-964. DOI=10.1145/1459359.1459533

Johnston, Andrew. 2009. Interfaces for Musical Expression Based on Simulated Physical Models. PhD diss., University of technology, Sydney

Laban, R., and Lawrence, F. C. Effort . London: MacDonald and Evans., 1947. 
Momeni, Ali and Cyrille Henry. 2006. "Dynamic independent mapping layers for concurrent control of audio and video synthesis." Computer Music Journal 30 (1): 49-66.

Puckette, Miller. 1998. "Real-time audio analysis tools for PD and MSP." in Proceedings, International Computer Music Conference, 109-112.

Spinuzzi, Clay. 2005. “The methodology of participatory design.” Technical Communication 52 (2): $163-$ 174.

Stam, Jos. 1999. "Stable fluids." in Proceedings of the 26th annual conference on Computer graphics and interactive techniques, ACM Press/Addison-Wesley Publishing Co., New York, NY, USA, 121128. DOI=10.1145/311535.311548

Verplank, W. L., M. V. Mathews, and R. Shaw. 2000. "Scanned Synthesis." in Proceedings of the International Computer Music Conference, 368-371.

\section{Bios}

Sarah Fdili Alaoui is postdoctoral researcher in the School of Interactive Arts and Technology at Simon Fraser University in Vancouver, Canada. Previously, she was a researcher at IRCAM, LIMSICNRS and University Paris-Sud. Her research concerns the study of dance gestures and their use in interaction design. She is involved in several collaborations with dancers and choreographers, to create interactive systems for dance performance and pedagogy. Sarah holds an engineering degree in Computer Science and Applied Mathematics from ENSIMAG and a Masters in Applied Mathematics from the University Joseph Fourier. She received a Ph.D. in Computer Science from the University Paris-Sud on the analysis and representation of dance gestures, where she studied the contribution of dance movement qualities in human-computer interaction. Sarah has also been trained in classical and contemporary dance and performed in contemporary dance companies and improvised dance collectives. Sarah is currently completing a qualification as a Certified Movement Analyst (CMA) at the Laban/Bartenieff Institute for Movement Studies (LIMS). She is interested in bridging scientific and experiential research in the movement based arts to radically alter the study of movement as a modality, strategy and method for transforming our understanding of human knowledge and cognition.

Cyrille Henry is working at the intersection of art, computer science and scientific research. His work incorporates the use of sensors, movement analysis, physical modeling, image processing as well as real time audio-visual synthesis. He previously worked as head of the hardware department of La Kitchen for four years (2001-2005) with the aim of creating sensor interfaces and to develop their uses in an artistic context (performing arts, interactive installation, contemporary music). Cyrille holds a Masters in Physics from the University du Maine. Since 2006, he has been independent developer and artist in the collective chdh. He collaborates with various dance companies and artists to create elaborate devices for digital installations and interactive stages.

Christian Jacquemin is a Professor in Computer Science at the University of Paris-Sud and coleads the Virtuality, Interaction, and Design and Art (VIDA) project at CNRS-LIMSI with Nathalie Delprat. From 1987 to 2000, he was working in Natural Language Processing. Since 2000, his work is focused on interactive 3D graphics and its applications for information visualization, virtual and mixed realities, and visual arts. He is involved in several collaborations on the artistic application of interactive 
graphics (theater, art installations, sound and graphic design...). His current research interests include information visualization (visual analytics), interactive 3D graphics and audio, advanced graphics rendering and applications to arts. He has collaborated with several artists and designers on the realization of augmented reality environments for art installation, theater plays and multimedia performances. He has published in major conferences in Computational Linguistics, Information Retrieval, Information Visualization, Multimedia, Digital and Performing arts. 